\title{
LHC tests of light neutralino dark matter without light sfermions
}

\author{
Lorenzo Calibbi, ${ }^{a}$ Jonas M. Lindert, ${ }^{b}$ Toshihiko Ota $^{c}$ and Yasutaka Takanishi ${ }^{d}$ \\ ${ }^{a}$ Service de Physique Théorique, Université Libre de Bruxelles, \\ Bld du Triomphe, CP225, B-1050 Brussels, Belgium \\ ${ }^{b}$ Physik-Institut, Universität Zürich, \\ Wintherturerstrasse 190, CH-8057 Zürich, Switzerland \\ ${ }^{c}$ Department of Physics, Saitama University, \\ Shimo-Okubo 255, 338-8570 Saitama-Sakura, Japan \\ ${ }^{d}$ Max-Planck-Institut für Kernphysik, \\ Saupfercheckweg 1, D-69117 Heidelberg, Germany \\ E-mail: lcalibbi@ulb.ac.be, lindert@physik.uzh.ch, toshi@mppmu.mpg.de, \\ yasutaka@mpi-hd.mpg.de
}

ABSTRACT: We address the question how light the lightest MSSM neutralino can be as dark matter candidate in a scenario where all supersymmetric scalar particles are heavy. The hypothesis that the neutralino accounts for the observed dark matter density sets strong requirements on the supersymmetric spectrum, thus providing an handle for collider tests. In particular for a lightest neutralino below $100 \mathrm{GeV}$ the relic density constraint translates into an upper bound on the Higgsino mass parameter $\mu$ in case all supersymmetric scalar particles are heavy. One can define a simplified model that highlights only the necessary features of the spectrum and their observable consequences at the LHC. Reinterpreting recent searches at the LHC we derive limits on the mass of the lightest neutralino that, in many cases, prove to be more constraining than dark matter experiments themselves.

KeYWords: Supersymmetry Phenomenology

ARXIV EPRINT: 1410.5730 


\section{Contents}

1 Introduction 1

2 Resonant neutralino annihilations 3

2.1 Constraints and viable parameter space $\quad 5$

$\begin{array}{lll}2.2 & \text { Direct and indirect DM searches } & 7\end{array}$

$\begin{array}{llr}3 & \text { LHC phenomenology } & 9\end{array}$

4 LHC limits $\quad 13$

5 Conclusions $\quad 15$

$\begin{array}{ll}\text { A Neutralino masses and mixing } & 16\end{array}$

\section{Introduction}

Employing the data collected at 7 and $8 \mathrm{TeV}$ of center of mass energy, the LHC experiments have recently published the results of an impressive number of searches for electroweak production of new physics. In many cases, they were able to set constraints on the masses of new electroweakly-interacting particles above the previous best bounds from LEP. This is the case in particular for the electroweak sector of the minimal supersymmetric standard model (MSSM), as well as of any of its extensions. The exact bounds depend on the details of the spectrum, especially on the mass hierarchy controlling the decay chains, and there is a generic loss of sensitivity in the regime of low mass splittings. However, it is remarkable that, in the most favourable cases, the limits in the MSSM are up to $300 \mathrm{GeV}$ for the sleptons $[1,2]$ and up to $700 \mathrm{GeV}$ for the charginos and neutralinos [2, 3].

The above mentioned searches have a crucial role in testing supersymmetric Dark Matter (DM) scenarios as they allow to probe the relevant parameter space independently of the colored sector of the theory, which might in principle be too heavy to be directly accessed by the LHC experiments. The cardinal idea is the following: the measurements of the DM relic density based on Cosmic Microwave Background (CMB) observations set nontrivial requirements on the supersymmetric spectrum, thus providing an handle for collider tests. This is true in particular if the lightest supersymmetric particle (LSP) is a bino-like neutralino, whose weak interactions typically lead to overproduction in the early universe, unless an efficient annihilation mechanism is at work. Since a limited set of supersymmetric particles and parameters is involved in the computation of the neutralino annihilation cross section, and hence of its relic density, one can define simplified models that highlight only the necessary features of the spectrum and their observable consequences at the LHC. 
The above sketched procedure has been recently employed by us to answer the question on how light the MSSM neutralino is still allowed to be by direct searches for electroweaklyinteracting supersymmetric particles at the LHC $[4,5]$. Other related studies on light neutralino Dark Matter have been recently published in [6-9]. For neutralinos lighter than about $30 \mathrm{GeV}$, the typical spectrum selected by the relic density constraints features rather light staus and Higgsinos, with masses smaller than few hundred GeV [10]. The electroweak production of these particles and the following decays lead to events with multiple taus and missing transverse momentum. Employing an ATLAS search for such a signature in combination with the limits on the decay rate of the Higgs into neutralinos, we could set a lower bound on the DM mass at about $24 \mathrm{GeV}$. Remarkably, with the above exercise, we showed that electroweak LHC searches are at the moment more powerful than direct and indirect searches in testing light neutralino Dark Matter. For early works addressing limits on light neutralino Dark Matter, see e.g. [11-13], and for limits on (very) light neutralinos without cosmological bounds we refer to refs. $[14,15]$ and references therein.

In the present paper, we want to extend our previous work to the case where no light sfermions are in the spectrum, i.e. scenarios with only neutralinos and charginos lighter than few hundreds GeV. A motivation for such an exercise is that light Higgsinos are the minimal 'tree-level' requirement posed by naturalness arguments. A Higgsino-like LSP can not however account for the full amount of the observed Dark Matter, unless its mass is in the $\mathrm{TeV}$ range, since the Higgsino-Higgsino annihilation processes are too efficient, see e.g. [16]. Simultaneous presence of light bino and Higgsinos is thus the minimal ingredient for electroweak scale neutralino Dark Matter in natural SUSY. Scenarios with mixed binoHiggsino Dark Matter, labelled as 'well-tempered neutralino', can provide a natural DM candidate overcoming the above mentioned problems of a pure Higgsino (or wino) LSP [17]. ${ }^{1}$ We are however interested to focus on the light DM regime (i.e. $m_{\widetilde{\chi}_{1}^{0}} \lesssim 100 \mathrm{GeV}$ ), where the neutralino can not be 'well-tempered' as it is bounded to be mainly bino due to chargino mass limits. Let us note in passing that, even giving up naturalness like in split SUSY scenarios [19, 20], or rather 'mini-split' [21] as suggested by the observed Higgs mass, the set-up we are studying is relevant to obtain the absolute lower bound on DM mass. In fact, in these models there are no light sfermions that can mediate the neutralino annihilation and the relic density requirements must be fulfilled by the gaugino-Higgsino sector alone.

As we are going to see, possible resonant enhancements of the neutralino annihilation cross section due to s-channel $Z$ and $h$ exchanges play a crucial role in the low mass regime we are going to study. This provides a further, purely phenomenological, motivation for our study: the effective coupling with nuclei for neutralino Dark Matter close to the above mentioned resonances might drastically drop, as well as the today annihilation rate relevant for indirect DM searches, hence one has to find alternative handles to test this corner of the parameter space. As we are going to show, if nature has chosen this peculiar scenario, LHC experiments compete and in some cases prove to be more constraining than dedicated DM experiments.

\footnotetext{
${ }^{1}$ For a recent discussion of the LHC prospects of this scenario, see [18].
} 
LHC limits and prospects for the gaugino-Higgsino sector of the MSSM have been recently discussed - however, without a focus on light neutralino DM - in [22-28], including the challenging case of compressed spectra.

The rest of the paper is organized as follows. In section 2 we present the light neutralino parameter space, where resonant annihilation dominates, including relevant collider and astrophysical constraints. In section 3 we discuss the resulting LHC phenomenology and in section 4 we present the corresponding limits. Finally, in section 5 we conclude.

\section{Resonant neutralino annihilations}

As anticipated in the introduction, we are interested to study the phenomenology of the MSSM neutralino as a Dark Matter candidate in the low-mass regime, i.e. with $m_{\widetilde{\chi}_{1}^{0}} \lesssim$ $100 \mathrm{GeV}$, in the case that only neutralinos and charginos are possibly light, while the rest of the spectrum, in particular the sfermions, might be decoupled. This setup is completely defined by the parameters that describe the gaugino-Higgsino sector in the MSSM:

$$
M_{1}, \quad M_{2}, \quad \mu, \quad \tan \beta,
$$

which are respectively the SUSY-breaking bino and wino masses, the superpotential Higgs mixing parameter that controls the spontaneous electroweak symmetry breaking and sets the mass of the Higgsinos, and the ratio of the two Higgs doublets vevs.

As a result of the LEP limit on charginos, $m_{\widetilde{\chi}_{1}^{ \pm}} \approx \min \left(M_{2},|\mu|\right) \gtrsim 100 \mathrm{GeV}$, the lightest neutralino has to be mainly bino in the mass range we consider. As usual, an efficient annihilation mechanism is thus required in order to satisfy the relic density constraints from CMB observations. Since we are assuming that there are no sfermions (and no extra Higgs bosons) below few hundreds $\mathrm{GeV}$ or more, the main annihilation modes go through an $s$-channel $Z$ or $h$ exchange:

$$
\widetilde{\chi}_{1}^{0} \widetilde{\chi}_{1}^{0} \rightarrow Z^{*} / h^{*} \rightarrow f \bar{f}
$$

Full expressions for the corresponding annihilation cross sections can be found in [29]. Let us recall here that the $s$-wave contribution vanishes in the $h$ mediation case and it is suppressed by a factor $m_{f}^{2} / m_{Z}^{2}$ for a $Z$ exchange. On the other hand, $p$-wave contributions are in both cases only suppressed by the temperature, $\sim T / m_{\widetilde{\chi}_{1}^{0}}$, and are therefore relevant for the calculation of annihilation rate in the early universe.

In order to have a qualitative understanding of the dependence of the relic density on the parameters shown in eq. (2.1), we have to consider the interactions of the neutralinos with $Z$ and $h$ only. They are given by the following expressions [30]:

$$
\begin{aligned}
\mathcal{L}_{\widetilde{\chi}_{i}^{0} \widetilde{\chi}_{j}^{0} Z} & =\frac{g}{2 c_{W}} Z_{\rho} \overline{\widetilde{\chi}_{i}^{0}} \gamma^{\rho}\left[O_{i j}^{Z L} \mathrm{P}_{L}+O_{i j}^{Z R} \mathrm{P}_{R}\right] \widetilde{\chi}_{j}^{0}, \\
\mathcal{L}_{\widetilde{\chi}_{i}^{0} \widetilde{\chi}_{j}^{0} h} & =\frac{g}{2} C_{i j}^{h} h \overline{\widetilde{\chi}_{i}^{0}} \widetilde{\chi}_{j}^{0},
\end{aligned}
$$

where the couplings are defined as:

$$
\begin{aligned}
O_{i j}^{Z L} & =-\frac{1}{2} N_{i 3} N_{j 3}^{*}+\frac{1}{2} N_{i 4} N_{j 4}^{*}, \quad O_{i j}^{Z R}=-O_{i j}^{Z L *} \\
C_{i j}^{h} & =\frac{1}{2}\left[\left(N_{i 2}-N_{i 1} \tan \theta_{W}\right)\left(\sin \alpha N_{j 3}+\cos \alpha N_{j 4}\right)+(i \leftrightarrow j)\right] .
\end{aligned}
$$


The matrix $N$ diagonalizes the neutralino mass matrix:

$$
\widetilde{\chi}_{i}^{0}=N_{i 1} \widetilde{B}+N_{i 2} \widetilde{W}^{0}+N_{i 3} \widetilde{H}_{d}^{0}+N_{i 4} \widetilde{H}_{u}^{0}
$$

We refer to the appendix for further details on our conventions and relevant approximate formulae for the elements $N_{i \alpha}$.

From the expressions in eqs. (2.3)-(2.6), we see that the couplings of the lightest neutralino to $Z$ and $h$ vanish if $\widetilde{\chi}_{1}^{0}$ is pure bino (or wino), i.e. if $N_{13}=N_{14}=0$. This only occurs when the Higgsino sector is decoupled, $\mu \gg M_{1}, m_{Z}$. In fact, using the approximate expressions shown in the appendix for the Higgsino components of $\widetilde{\chi}_{1}^{0}$, we find:

$$
N_{13}=\frac{m_{Z} s_{W}}{\mu}\left[s_{\beta}+c_{\beta} \frac{M_{1}}{\mu}\right], \quad N_{14}=-\frac{m_{Z} s_{W}}{\mu}\left[c_{\beta}+s_{\beta} \frac{M_{1}}{\mu}\right]
$$

where for simplicity we assumed $M_{2} \gg|\mu|$. Here we defined $c_{\beta} \equiv \cos \beta, s_{\beta} \equiv \sin \beta$ and $s_{W} \equiv \sin \theta_{W}$. It is therefore clear that the upper limit on the DM relic density will translate into an upper limit on $|\mu|$, i.e. on the mass scale of the Higgsinos. Thus, relatively light Higgsinos are a generic prediction of our setup, while from eqs. (2.5), (2.6) it is clear that the wino plays no crucial role in the annihilation process and might in principle be heavier.

A closer look at the expressions for the annihilation cross section reported in [29] shows the well-known possibility of a resonant enhancement of the $p$-wave annihilation, occurring if

$$
m_{\widetilde{\chi}_{1}^{0}} \approx m_{Z} / 2 \quad \text { or } \quad m_{\widetilde{\chi}_{1}^{0}} \approx m_{h} / 2
$$

Obviously, the closer $m_{\widetilde{\chi}_{1}^{0}}$ approaches these conditions the looser the upper bound on $\mu$ becomes, since the enhancement can compensate smaller couplings of $\widetilde{\chi}_{1}^{0} \widetilde{\chi}_{1}^{0} Z, \widetilde{\chi}_{1}^{0} \widetilde{\chi}_{1}^{0} h$. On the other hand, we expect the relic density constraints to set a tighter bound on $\mu$ as the mass $m_{\widetilde{\chi}_{1}^{0}}$ lies further from the resonant conditions of eq. (2.9). In what follows, we illustrate and quantify these simple features by means of a numerical scan of the relevant parameters (in section 2.1) and we discuss the LHC phenomenology of this region of resonant neutralino dark matter (in section 3) and the constraints set by searches for chargino-neutralino production (in section 4 ).

Before moving on, let us comment about the possible role of the extra Higgses. It is well known, that an $s$-channel exchange of the CP-odd Higgs $A$ can also provide an efficient annihilation mechanism for neutralino DM, especially close to the resonant condition $m_{\widetilde{\chi}_{1}^{0}} \approx$ $m_{A} / 2$ and/or for a sizeable Higgsino component in $\widetilde{\chi}_{1}^{0}$ [31]. However, in the light neutralino regime we are considering, $A$ would be required to be relatively light $[13,32-34]$. This possibility is challenged [35] by direct searches for extra Higgses at the LHC [36], as well as by the measurements of the Higgs production and decays, that prove to be SM-like at least at the $30 \%$ level (see e.g. [37]), and by rare decays such as $B_{s} \rightarrow \mu^{+} \mu^{-}$[38]. For these reasons, here we do not consider the possibility that the extended Higgs sector of the MSSM plays a role in the neutralino annihilation and we assume for simplicity that the heavy Higgses are also decoupled. 

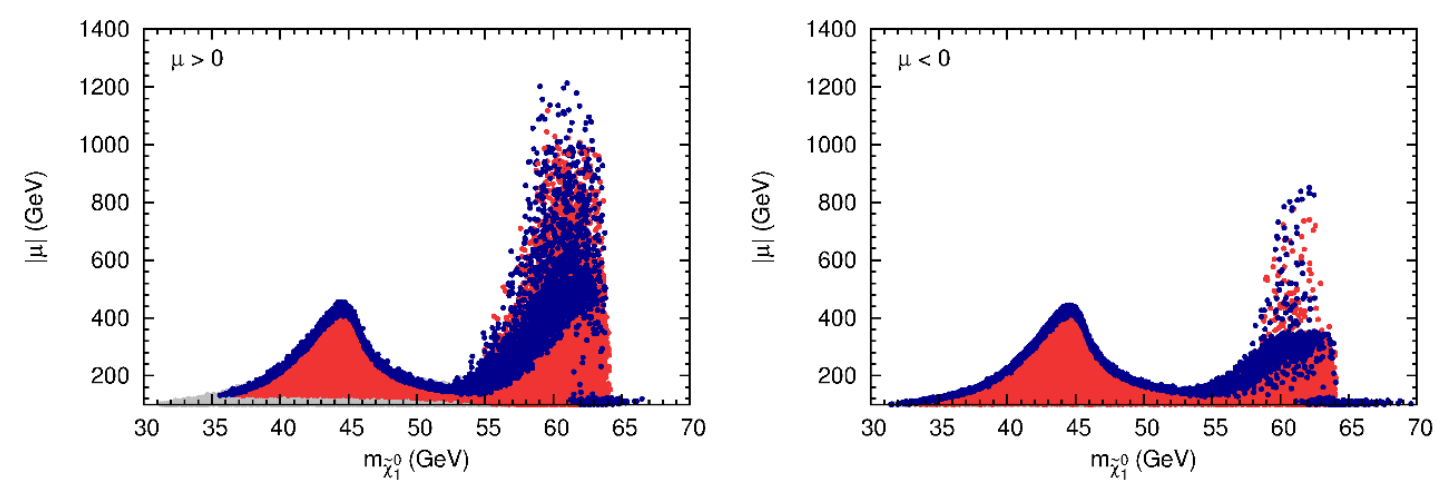

Figure 1. Results of the parameter scan defined in eqs. (2.10)-(2.12) in the $m_{\tilde{\chi}_{1}^{0}}-|\mu|$ plane for $\mu>0$ (left panel), $\mu<0$ (right panel). Red points satisfy the relic density upper bound of eq. (2.13) and all other constraints discussed in the text. Blue points in addition satisfy the lower bound. Gray points are excluded by one of the constraints listed in the text.

\subsection{Constraints and viable parameter space}

Here we present the results of a random scan of our four parameters within the following ranges:

$$
\begin{aligned}
20 \mathrm{GeV} & \leq M_{1} \leq 80 \mathrm{GeV}, & 100 \mathrm{GeV} & \leq M_{2} \leq 1 \mathrm{TeV} \\
100 \mathrm{GeV} & \leq|\mu| \leq 1 \mathrm{TeV}, & 5 & \leq \tan \beta \leq 50 .
\end{aligned}
$$

Notice that we scan both signs of $\mu$ while we take $M_{i}>0$ with no loss of generality: observable effects depend in fact on the relative $\operatorname{sign} \operatorname{sgn}\left(\mu M_{i}\right)$. Furthermore we vary the soft parameters of the stop sector in the following ranges:

$$
2 \mathrm{TeV}<m_{\widetilde{t}_{L}}, m_{\widetilde{t}_{R}} \leq 5 \mathrm{TeV} \mathrm{GeV}, \quad-4 \mathrm{TeV} \leq A_{t}<4 \mathrm{TeV} .
$$

Together with the ones in eq. (2.10) these parameters determine the value of the physical Higgs mass $m_{h}$ and thus of the position of the resonance in eq. (2.9). The other SUSY soft parameters were set to the following constant values:

$$
m_{\widetilde{f}}=M_{3}=m_{A}=4 \mathrm{TeV}, A_{f}=0,
$$

where $m_{\widetilde{f}}$ represents the remaining sfermion masses, $M_{3}$ is the gluino mass, $m_{A}$ the CP-odd Higgs mass, $A_{f}$ the remaining trilinear couplings. The spectrum has been computed by means of the routine SuSpect [39], the branching fractions by the SUSY-HIT package [40] and micrOMEGAs [41-44] has been used to calculate the neutralino relic density, as well as the scattering cross section with nuclei and the present thermally-averaged annihilation cross section.

The constraints we impose on our parameter space are presented in the following.

- DM relic density. We assume a standard thermal history of the universe and take this conservative range from ref. [45]:

$$
0.10 \leq \Omega_{\mathrm{DM}} h^{2} \leq 0.13 .
$$


- Direct SUSY searches at LEP. The 95\% CL bound on the lightest chargino mass is

$$
m_{\widetilde{\chi}_{1}^{ \pm}} \geq 94 \mathrm{GeV}
$$

Searches for $\widetilde{\chi}_{1}^{0} \widetilde{\chi}_{2,3}^{0}$ associated production at LEP, followed by the decay $\widetilde{\chi}_{2,3}^{0} \rightarrow$ $\widetilde{\chi}_{1}^{0} Z^{(*)}$, set a constraint for $m_{\widetilde{\chi}_{1}^{0}}+m_{\widetilde{\chi}_{2,3}^{0}} \geq \sqrt{s}=208 \mathrm{GeV}$. This conservatively reads:

$$
\sum_{k=2,3} \sigma\left(e^{+} e^{-} \rightarrow \widetilde{\chi}_{1}^{0} \widetilde{\chi}_{k}^{0}\right) \times \operatorname{BR}\left(\widetilde{\chi}_{k}^{0} \rightarrow \widetilde{\chi}_{1}^{0} Z^{(*)}\right)<100 \mathrm{fb}
$$

We estimated the production cross sections at LEP using the leading order formulae reported in refs. [46, 47].

- $Z$ invisible width. As discussed above, the relic density constraint require sizeable $\widetilde{\chi}_{1}^{0} \widetilde{\chi}_{1}^{0} Z$ and $\widetilde{\chi}_{1}^{0} \widetilde{\chi}_{1}^{0} h$ couplings. As a consequence, the invisible decays $Z \rightarrow \widetilde{\chi}_{1}^{0} \widetilde{\chi}_{1}^{0}$ and $h \rightarrow \widetilde{\chi}_{1}^{0} \widetilde{\chi}_{1}^{0}$ can occur at relevant rates if kinematically allowed. The decay width of the $Z$ boson into a neutralino pair is given by [48]:

$$
\Gamma\left(Z \rightarrow \widetilde{\chi}_{1}^{0} \widetilde{\chi}_{1}^{0}\right)=\frac{G_{F} m_{Z}^{3}}{12 \sqrt{2} \pi}\left(1-\frac{4 m_{\widetilde{\chi}_{1}^{0}}^{2}}{m_{Z}^{2}}\right)^{\frac{3}{2}}\left|N_{13}^{2}-N_{14}^{2}\right|^{2},
$$

This has to be compared to the LEP bound on the new physics contribution to $\Gamma(Z \rightarrow$ invisible $)[49]:$

$$
\Delta \Gamma_{Z}^{\mathrm{inv}}<3 \mathrm{MeV} \quad(95 \% \mathrm{CL})
$$

- Higgs mass and rates. Applying the tools HiggsBounds [50-52] and HiggsSignals [53] we calculate a $\chi^{2}$ measure for the predictions of the model and the measured Higgs rates and mass. We ensure an agreement between the predicted light Higgs mass and production rates and the current experimental measurements at the 95\% CL requiring a p-value below 0.002 .

- Invisible Higgs decays. The light Higgs decay width into $\widetilde{\chi}_{1}^{0} \widetilde{\chi}_{1}^{0}$ is given by [54]:

$$
\Gamma\left(h \rightarrow \widetilde{\chi}_{1}^{0} \widetilde{\chi}_{1}^{0}\right)=\frac{\sqrt{2} G_{F} m_{W}^{2} m_{h}}{\pi}\left(1-\frac{4 m_{\tilde{\chi}_{1}^{0}}^{2}}{m_{h}^{2}}\right)^{\frac{3}{2}}\left|C_{11}^{h}\right|^{2},
$$

where from eq. (2.6) one finds in the decoupling regime $m_{A} \gg m_{h}$ :

$$
C_{11}^{h}=\frac{1}{2}\left(N_{12}-\tan \theta_{W} N_{11}\right)\left(\sin \beta N_{14}-\cos \beta N_{13}\right) .
$$

Since a sizeable $\Gamma(h \rightarrow$ invisible) would reduce by the same amount the branching fractions of all visible channels, it can be constrained by fits to the observed Higgs decay rates. In this work we adopt the limit reported in [37]:

$$
\mathrm{BR}(h \rightarrow \text { invisible }) \lesssim 26 \% \quad(95 \% \mathrm{CL}) .
$$



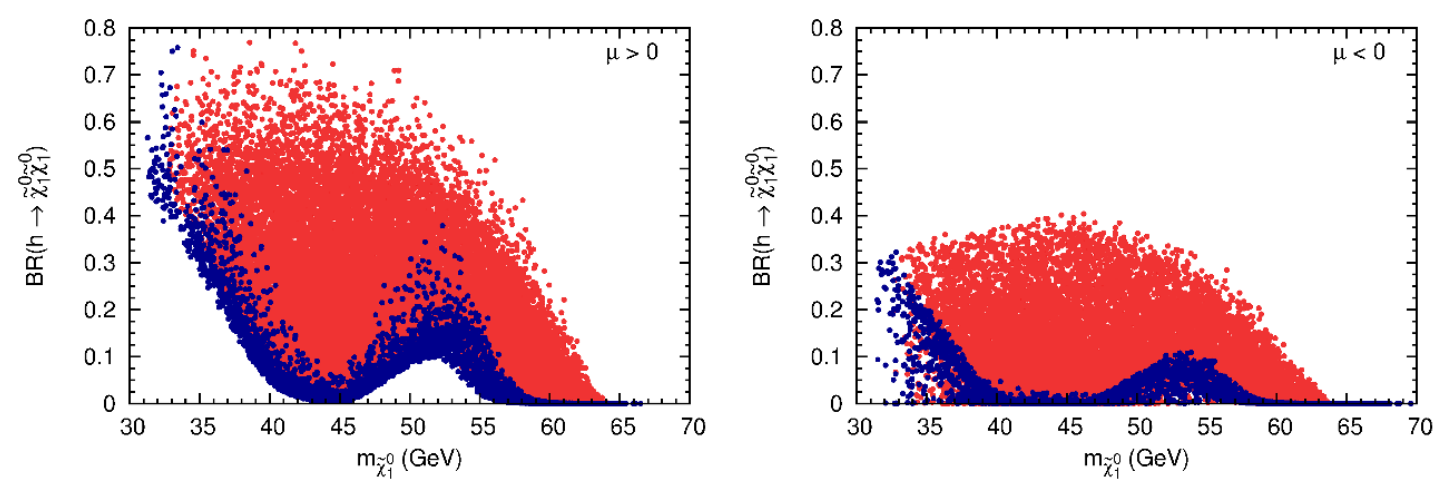

Figure 2. Predictions for the invisible branching ratio of the Higgs $\operatorname{BR}\left(h \rightarrow \widetilde{\chi}_{1}^{0} \widetilde{\chi}_{1}^{0}\right)$ in the paramter scan defined in eqs. (2.10)-(2.12) for $\mu>0$ (left panel), $\mu<0$ (right panel). Red points satisfy the relic density upper bound of eq. (2.13) and all other constraints discussed in the text. Blue points in addition satisfy the lower bound.

Possible further constraints from electroweak precision observables or the flavour sector can be circumvented adjusting the parameters in eq. (2.12).

In figure 1 we show the results of the parameter scan in the plane of $m_{\widetilde{\chi}_{1}^{0}}$ against the Higgsino mass parameter $\mu$ for both signs of $\mu$. The red points only fulfill the upper bound of eq. (2.13), while the blue ones fulfill the lower bound too. Thus the blue points correspond to models where $\widetilde{\chi}_{1}^{0}$ can account for $100 \%$ of the observed Dark Matter. Points excluded by any of the constraints explained above but the relic density constraint are shown in grey and - marginalizing over all other parameters - they affect the parameter space only for $\mu>0$ at small values of $\mu$.

As argued already in the previous section, if $m_{\widetilde{\chi}_{1}^{0}}$ is slightly away from the resonances, eq. (2.13) tightly constrains $\mu$. On the other hand, Higgsinos can be as heavy as $\approx 450 \mathrm{GeV}$ close to the $Z$-pole and as heavy as $\approx 1200(900) \mathrm{GeV}$ close to the $h$ resonance for $\mu>0$ $(\mu<0)$. The width and shape of the Higgs resonance is determined by the possible spread in the Higgs mass. Clearly, the parameter region very close to the $h$ resonance is difficult to cover entirely at the LHC.

For illustration in figure 2, we explicitly show the invisible branching ratio of the Higgs $\operatorname{BR}\left(h \rightarrow \widetilde{\chi}_{1}^{0} \widetilde{\chi}_{1}^{0}\right)$ for both signs of $\mu$ obtained in our parameter scan. All constraints but the one from the invisible width of the Higgs itself are applied and the color-coding is as in figure 1. As we can see, eq. (2.20) excludes points for $m_{\widetilde{\chi}_{1}^{0}} \lesssim 35 \mathrm{GeV}$ if $\mu>0$. For $\mu<0$ no such limit can be obtained. In fact, as one can see from eq. (2.8), a partial cancellation in the $\widetilde{\chi}_{1}^{0} \widetilde{\chi}_{1}^{0} h$ vertex decreases the coupling if there is a relative sign between $\mu$ and $M_{1}$. This is also the reason why smaller values of $|\mu|$ are required close to the $h$ resonance for $\mu<0$, see figure 1 . We want to note that for the considered parameter space, regions excluded from the invisible width of the Higgs encompass exclusions from all other constraints considered here.

\subsection{Direct and indirect DM searches}

As sketched in the introduction, direct and indirect DM searches can loose their sensitivity in the vicinity of the resonant annihilation regimes, eq. (2.9). We quantify this behaviour in 

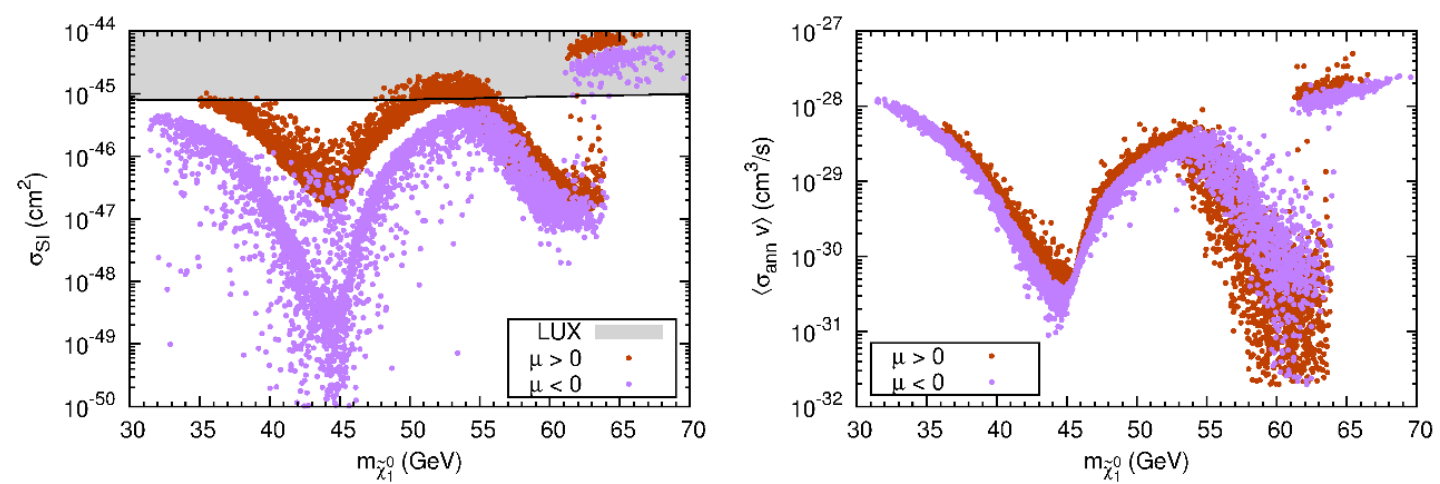

Figure 3. Predictions for the spin-independent DM-nucleon scattering cross section $\sigma_{\mathrm{SI}}$ (left panel) and the present thermally-averaged annihilation cross section $\left\langle\sigma_{\mathrm{ann}} v\right\rangle$ (right panel) in the paramter scan defined in eqs. (2.10)-(2.12). All points satisfy the upper and lower bound of eq. (2.13) and all other constraints discussed in section 2.1. The gray shaded area in the left plot is excluded from direct DM searches with LUX.

figure 3, where the spin-independent DM-nucleon scattering cross section $\sigma_{\mathrm{SI}}$ (left panel) and the present thermally-averaged annihilation cross section $\left\langle\sigma_{\mathrm{ann}} v\right\rangle$ (right panel) are plotted as a function of the lightest neutralino mass for the points of our parameter scan defined in eqs. (2.10)-(2.12). All shown points account for the observed DM abundance, i.e. they satisfy the upper and lower bound of eq. (2.13) besides all other constraints discussed in section 2.1. Red (purple) points correspond to $\mu>0(\mu<0)$.

In the left panel, we show as a reference the current limit set by the direct search experiment LUX [55], which for the considered mass range is almost independent of the neutralino mass at $\sigma_{\mathrm{SI}} \lesssim 8 \times 10^{-46} \mathrm{~cm}^{2}$. Close to the resonances the predicted $\sigma_{\mathrm{SI}}$ is suppressed by several orders of magnitude and tests of such scenarios even in future direct DM search experiments seems to be very challenging. The neutralino elastic scattering with nuclei is mediated by the exchange of CP-even Higgs states or squarks (which we assume to be decoupled). Thus, the shown suppression originates from small Higgs-Higgsinobino couplings, as given in eq. (2.6), close to the resonances (due to large $\mu$ as required by the relic abundance). Larger values of this coupling, i.e. a smaller $\mu$ parameter would reduce the neutralino density $\Omega_{\widetilde{\chi}^{0}}$ below the observed value, which would require extra DM components and, more importantly for us, anyway would reduce the sensitivity of direct detection by a factor $\Omega_{\widetilde{\chi}^{0}} / \Omega_{\mathrm{DM}}^{\mathrm{obs}}$. However, we have to keep in mind that the theoretical prediction for $\sigma_{\mathrm{SI}}$ suffers from large uncertainties: variations of light quark masses and hadronic form factors, as well as heavier values of $m_{H} \approx m_{A}$ (here we took $m_{A}=4 \mathrm{TeV}$ ) can further reduce the predicted $\sigma_{\mathrm{SI}}$ by a factor of few. On the other hand, lighter heavy Higgs states, i.e. smaller values of $m_{A}$ could in principle increase the spin-independent cross section without altering much the relic density prediction. Therefore, we refrain from setting any conservative constraints on our parameter space from direct detection.

Similarly to the discussion above, we observe in the right panel of figure 3 that the predicted $\left\langle\sigma_{\text {ann }} v\right\rangle$ is well below the sensitivity of indirect detection experiments - which are currently at the level of $10^{-26} \mathrm{~cm}^{3} / \mathrm{s}$ [56] — and further drops in the vicinity of the 
resonances. The reason why the present $\left\langle\sigma_{\text {ann }} v\right\rangle$ is much lower than the value required by a thermal WIMP at the freeze-out can be understood by the following: at high temperatures the annihilation is dominated by resonant $p$-wave contributions which become irrelevant as the temperature drops. In the present universe, the annihilation occurs through a $Z$ mediated $s$-wave amplitude. The corresponding cross section is suppressed by a factor $m_{f}^{2} / m_{Z}^{2}$. Furthermore, as above, close to the resonances a small Higgsino component in $\widetilde{\chi}_{1}^{0}$ further suppresses the $\widetilde{\chi}_{1}^{0} \widetilde{\chi}_{1}^{0} Z$ coupling. Again an additional possible contribution to $\left\langle\sigma_{\text {ann }} v\right\rangle$ is expected to be provided by an $s$-channel exchange of a CP-odd Higgs $A$. We checked that even for masses at the border of the present LHC exclusion, e.g. $m_{A} \simeq 500 \mathrm{GeV}$ for $\tan \beta=20[36],\left\langle\sigma_{\mathrm{ann}} v\right\rangle$ can not increase by more than one order of magnitude with respect to the values shown in figure 3 .

\section{LHC phenomenology}

The spectrum we consider solely involves the neutralino/chargino sector of the MSSM. As discussed above, the relic density constraint translates into an upper bound on the Higgsino mass parameter $\mu$, while the wino mass parameter $M_{2}$ does hardly play a role satisfying those bounds. Thus, the minimal particle content are just the mostly bino-like neutralino LSP and the Higgsino states: two heavier neutralinos and the lightest charginos. Later we will demonstrate that additional light winos just increase the LHC sensitivity. Hence, taking $M_{2}$ to be large is a conservative assumption and will be assumed if not otherwise stated. All other SUSY particles are assumed to be decoupled.

For the described spectrum possible tests at the LHC rely on electroweak Drell-Yan production of the Higgsino-like states: ${ }^{2}$

$$
p p \rightarrow \widetilde{\chi}_{k}^{0} \widetilde{\chi}_{l}^{0}, \quad p p \rightarrow \widetilde{\chi}_{1}^{+} \widetilde{\chi}_{1}^{-}, \quad p p \rightarrow \widetilde{\chi}_{1}^{ \pm} \widetilde{\chi}_{k}^{0}, \quad(k, l=2,3) .
$$

The produced charginos can only decay into the LSP and (on- or off-shell) $W$ bosons:

$$
\widetilde{\chi}_{1}^{ \pm} \rightarrow W^{ \pm(*)} \widetilde{\chi}_{1}^{0}
$$

whereas the neutralinos have two competing decay modes, $Z$ or $h$ :

$$
\widetilde{\chi}_{2,3}^{0} \rightarrow Z^{(*)} \widetilde{\chi}_{1}^{0}, \quad \widetilde{\chi}_{2,3}^{0} \rightarrow h^{(*)} \widetilde{\chi}_{1}^{0}
$$

with relevance depending on the model parameters as discussed in the following.

The most relevant searches for neutralino/chargino production performed by the LHC collaborations are based on leptonic decays of the gauge bosons, i.e. on events with multiple leptons plus missing transverse momentum. For the parameter space we consider by far the highest sensitivity is reached in the $W Z$-channel [28] (from associated neutralino-chargino production, $\left.\widetilde{\chi}_{1}^{ \pm} \widetilde{\chi}_{2,3}^{0}\right)$ with three reconstructed leptons in the final state $[2,3]$. In this channel the search performed by ATLAS [3] sets the most stringent limits. Searches for the Higgs decay have been performed in the $W h$-channel with $h \rightarrow b \bar{b}[2]$, see also [24, 59]. The

\footnotetext{
${ }^{2}$ Monojet searches for direct production of a pair of neutralino LSPs in association with a jet can in principle also test the given spectrum but will only become sensitive in the future $[57,58]$.
} 

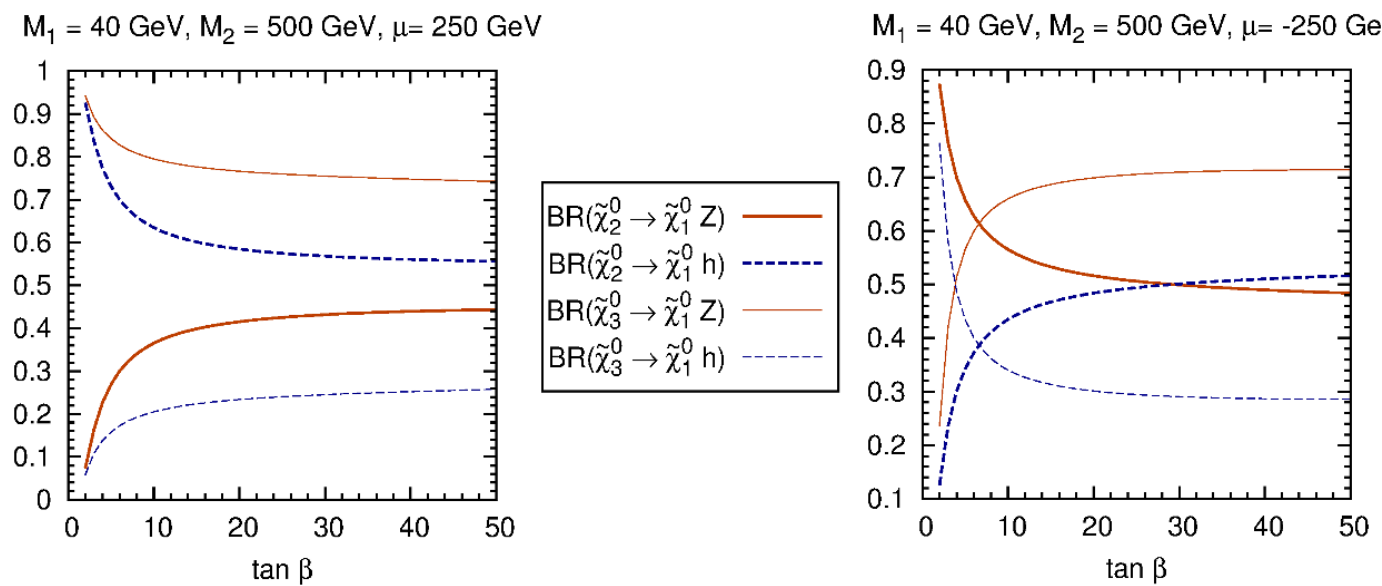

Figure 4. Branching ratios for the decay $\widetilde{\chi}_{2,3}^{0} \rightarrow \widetilde{\chi}_{1}^{0} Z / h$ as function of $\tan \beta$. Parameters are chosen to be $M_{1}=40 \mathrm{GeV}, M_{2}=500 \mathrm{GeV}$ and $\mu= \pm 250 \mathrm{GeV}$ for the left/right plot.

complementary $h \rightarrow \tau^{+} \tau^{-}$channel might yield a similar sensitivity [60]. However, the overall sensitivity in the $W h$-channel is considerably weaker compared to the $W Z$-channel. Still, it is in order to investigate in detail the rates of the competing decay modes shown in eq. (3.3). The decay rates of $\widetilde{\chi}_{2,3}^{0} \rightarrow Z \widetilde{\chi}_{1}^{0}$ are controlled by the couplings defined in eq. (2.5). Using the approximate expressions for the neutralino mixing in the Higgsino-like $\widetilde{\chi}_{2,3}^{0}$ limit $M_{2} \gg|\mu|$, as reported in the appendix eq. (A.7), we find:

$$
\begin{aligned}
& \mu>0: \quad O_{21}^{Z L} \simeq \frac{m_{Z} s_{W}}{2 \sqrt{2} \mu}\left(s_{\beta}-c_{\beta}\right)\left(1+\frac{M_{1}}{\mu}\right), \quad O_{31}^{Z L} \simeq \frac{m_{Z} s_{W}}{2 \sqrt{2} \mu}\left(s_{\beta}+c_{\beta}\right)\left(1+\frac{M_{1}}{\mu}\right) \\
& \mu<0: \quad O_{21}^{Z L} \simeq-\frac{m_{Z} s_{W}}{2 \sqrt{2} \mu}\left(s_{\beta}+c_{\beta}\right)\left(1+\frac{M_{1}}{\mu}\right), \quad O_{31}^{Z L} \simeq \frac{m_{Z} s_{W}}{2 \sqrt{2} \mu}\left(c_{\beta}-s_{\beta}\right)\left(1+\frac{M_{1}}{\mu}\right),
\end{aligned}
$$

Analogous expressions for the coupling $\widetilde{\chi}_{2,3}^{0} \widetilde{\chi}_{1}^{0} h$ can be obtained from eqs. (2.6), (A.7):

$$
\begin{aligned}
\mu>0: & C_{21}^{h} \simeq-\frac{1}{2 \sqrt{2}}\left(c_{\beta}+s_{\beta}\right), & C_{31}^{h} \simeq-\frac{1}{2 \sqrt{2}}\left(c_{\beta}-s_{\beta}\right) ; \\
\mu<0: \quad & C_{21}^{h} \simeq-\frac{1}{2 \sqrt{2}}\left(c_{\beta}-s_{\beta}\right), & C_{31}^{h} \simeq-\frac{1}{2 \sqrt{2}}\left(c_{\beta}+s_{\beta}\right) .
\end{aligned}
$$

From these expressions we expect that for $\mu>0$ the branching ratio $\operatorname{BR}\left(\widetilde{\chi}_{2}^{0} \rightarrow Z \widetilde{\chi}_{1}^{0}\right)$ decreases for small values of $\tan \beta$, and vanishes in the limit $\tan \beta \rightarrow 1$. Whereas the branching ratio $\operatorname{BR}\left(\widetilde{\chi}_{3}^{0} \rightarrow Z \widetilde{\chi}_{1}^{0}\right)$ is maximized in the low $\tan \beta$ regime. This behaviour is depicted in the left panel of figure 4, where for illustration we choose $M_{1}=40 \mathrm{GeV}, M_{2}=$ $500 \mathrm{GeV}$ and $\mu=250 \mathrm{GeV}$. The behaviour described above is reversed for $\mu<0$, as shown in the right panel of figure 4. Clearly, the $W Z$ channel is expected to suffer a loss of sensitivity in the low (large) $\tan \beta$ regime for $\mu>0(\mu<0)$. However, as the 


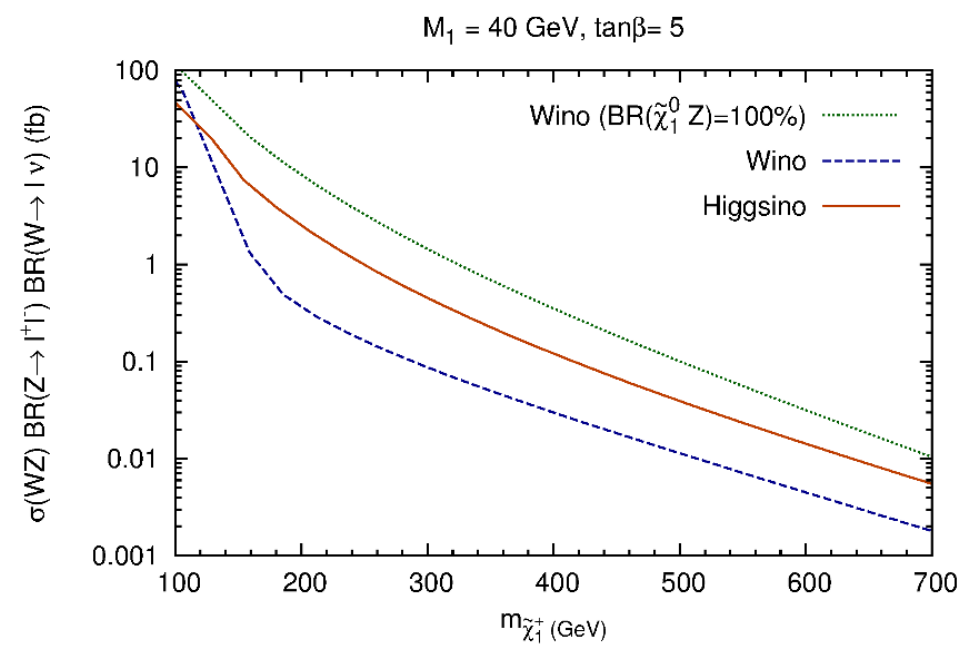

Figure 5. Comparison among the simplified wino model with $\operatorname{BR}\left(\widetilde{\chi}_{2}^{0} \rightarrow Z \widetilde{\chi}_{1}^{0}\right)=100 \%$, a realistic wino model with $M_{2} \ll \mu$ and an Higgsino model with $\mu \ll M_{2}$. Shown is the summed neutralinochargino production cross section times branching ratios into $W\left(\rightarrow \ell^{ \pm} \nu\right) Z\left(\rightarrow \ell^{+} \ell^{-}\right)$as defined in eq. (3.8).

behavior of the two Higgsino-like neutralinos $\widetilde{\chi}_{2,3}^{0}$ is antipodal ${ }^{3}$ and their mass splitting is in general small, the $\tan \beta$ dependence in the total sensitivity of the $W Z$ channel is moderate. In our numerical analysis in section 4 we consider the two example values $\tan \beta=5,40$. Furthermore, as the summed contribution only mildly depend on $\tan \beta$ even for small values of $\tan \beta$ the $W Z$-channel is expected to remain more sensitive than the Wh-channel.

Searching for neutralino and chargino production in the three leptons plus missing energy final state performed by ATLAS [3] the strongest available limits for the $W Z$ channel have been obtained. These limits have been interpreted in the $M_{2}-\mu$ plane of the pMSSM for fixed values of $M_{1}$ and in terms of constraints on the mass of purely wino-like charginos and neutralinos with $\operatorname{BR}\left(\widetilde{\chi}_{2}^{0} \rightarrow \widetilde{\chi}_{1}^{0} Z\right)=100 \%$. Clearly, the latter is only a simplified model as for a pure wino-like $\widetilde{\chi}_{2}^{0}$ state the coupling $\widetilde{\chi}_{1}^{0} \widetilde{\chi}_{2}^{0} Z$ vanishes. The corresponding limits for a realistic scenario with Higgsino-like neutralinos and charginos might be much weaker due to changes in the cross section and possible competing decay modes as discussed above. Therefore, in section 4 we will reinterpret those limits for Higgsino-like neutralinos in a detailed analysis including detector effects. Here, we already want to anticipate those results qualitatively. To this end we compare the production cross section times branching ratio for the $W Z$ channel defined as

$$
\begin{aligned}
\sigma_{3 \ell+E_{T}}=\sum_{\substack{k=1,2 \\
l=2,3,4}} \sigma\left(\widetilde{\chi}_{k}^{ \pm} \widetilde{\chi}_{l}^{0}\right) \operatorname{BR}\left(\widetilde{\chi}_{k}^{ \pm}\right. & \left.\rightarrow W^{ \pm} \widetilde{\chi}_{1}^{0}\right) \operatorname{BR}\left(\widetilde{\chi}_{l}^{0} \rightarrow Z \widetilde{\chi}_{1}^{0}\right) \times \\
& \times \mathrm{BR}\left(W^{ \pm} \rightarrow \ell^{ \pm} \nu\right) \mathrm{BR}\left(Z \rightarrow \ell^{+} \ell^{-}\right),
\end{aligned}
$$

\footnotetext{
${ }^{3}$ Also the production cross sections depend on $\tan \beta$ via the $\widetilde{\chi}_{2,3}^{0} \widetilde{\chi}_{1}^{ \pm} Z$ couplings. For fixed physical masses the dependence is very mild and again antipodal to the corresponding dependence in the branching ratios.
} 


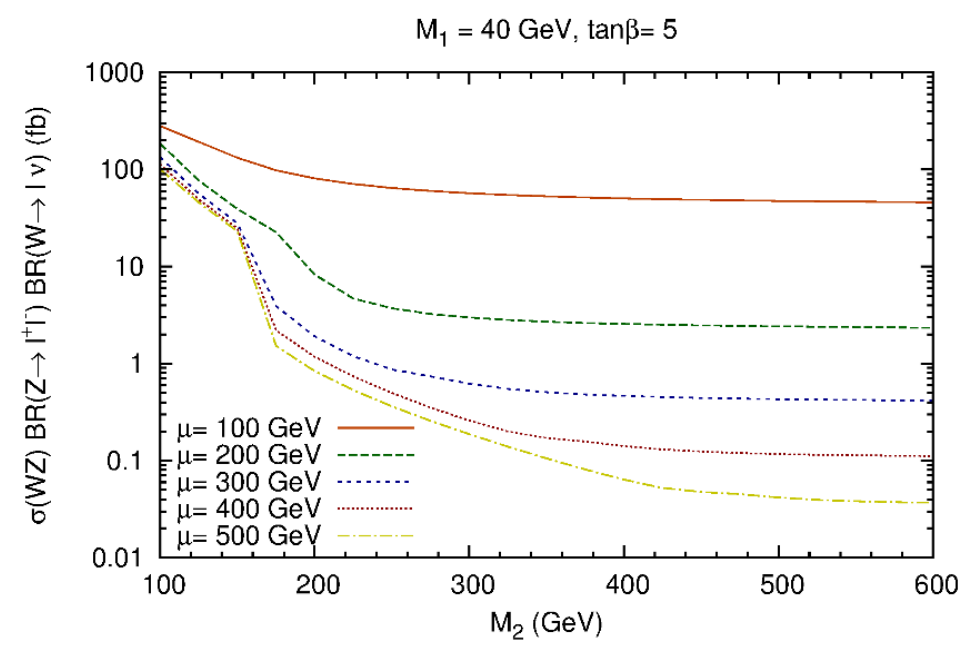

Figure 6. Impact of $M_{2}$ on the summed neutralino-chargino production cross section times branching ratios into $W\left(\rightarrow \ell^{ \pm} \nu\right) Z\left(\rightarrow \ell^{+} \ell^{-}\right)$as defined in eq. (3.8) for different values of $\mu$.

for the cases of (i) the simplified model, (ii) realistic wino-like $\widetilde{\chi}_{2}^{0}$ case, and (iii) Higgsino-like $\widetilde{\chi}_{2,3}^{0}$ case. The corresponding estimated rates are shown in figure 5 as a function of the mass of $\widetilde{\chi}_{1}^{ \pm}$, where (leading order) production cross sections are calculated with Prospino 2 [61] and BRs with SUSY-HIT [40]. In the Higgsino-like $\widetilde{\chi}_{2,3}^{0}$ case, we set $M_{2}=1 \mathrm{TeV}$ and, vice versa, we set $\mu=1 \mathrm{TeV}$ in the wino-like $\widetilde{\chi}_{2}^{0}$ cases. For all scenarios we additionally set for illustration $M_{1}=40 \mathrm{GeV}$ and $\tan \beta=5$. Clearly, for realistic values of $\operatorname{BR}\left(\widetilde{\chi}_{2}^{0} \rightarrow Z \widetilde{\chi}_{1}^{0}\right)$ compared to the simplified model the sensitivity is strongly reduced in the wino-like $\widetilde{\chi}_{2}^{0}$ case. Resulting rates are here also considerably smaller than in the Higgsino-like $\widetilde{\chi}_{2,3}^{0}$ case - despite the fact that the production cross section for wino states is typically larger than the one for Higgsinos. In [3] ATLAS obtains (approximately) the limit $m_{\widetilde{\chi}_{1}^{ \pm}} \gtrsim 350 \mathrm{GeV}$ for $m_{\widetilde{\chi}_{1}^{0}} \lesssim 100 \mathrm{GeV}$ under the assumption of $\operatorname{BR}\left(\widetilde{\chi}_{2}^{0} \rightarrow Z \widetilde{\chi}_{1}^{0}\right)=100 \%$. Comparing such a limit in figure 5 to the Higgsino-like $\widetilde{\chi}_{2,3}^{0}$ case we expect an exclusion on $m_{\widetilde{\chi}_{1}^{ \pm}} \approx m_{\widetilde{\chi}_{2}^{0}} \approx m_{\widetilde{\chi}_{3}^{0}}$ weaker by about $100 \mathrm{GeV}$. Still, this represents a non-negligible constraint on the neutralino DM parameter space.

Finally, let us turn to a short discussion of the possible impact of the wino mass $M_{2}$ on the signal rates in the $W Z$ channel. For $M_{2} \lesssim|\mu|$, the full set of neutralinos and charginos contributes to the production cross section, providing additional modes to those shown in eq. (3.1). Hence it is natural to expect an increase in sensitivity. This is confirmed and quantified in figure 6 , where we plot again cross section times branching ratio, as defined in eq. (3.8), now as a function of $M_{2}$ for different choices of $\mu$. In figure 6 we see that the number of expected leptonic events generically increases for low values of $M_{2}$, whereas it becomes approximately flat for $M_{2}>\mu$. As a consequence, fixing $M_{2}$ at some value larger than $\mu$ can be regarded as a conservative choice. We are going to adopt this choice in the numerical simulation of the next section.

Let us note here that further MSSM parameters, besides those of the neutralino/chargino sector, cf. eq. (2.1), have in general little impact on the searches based 


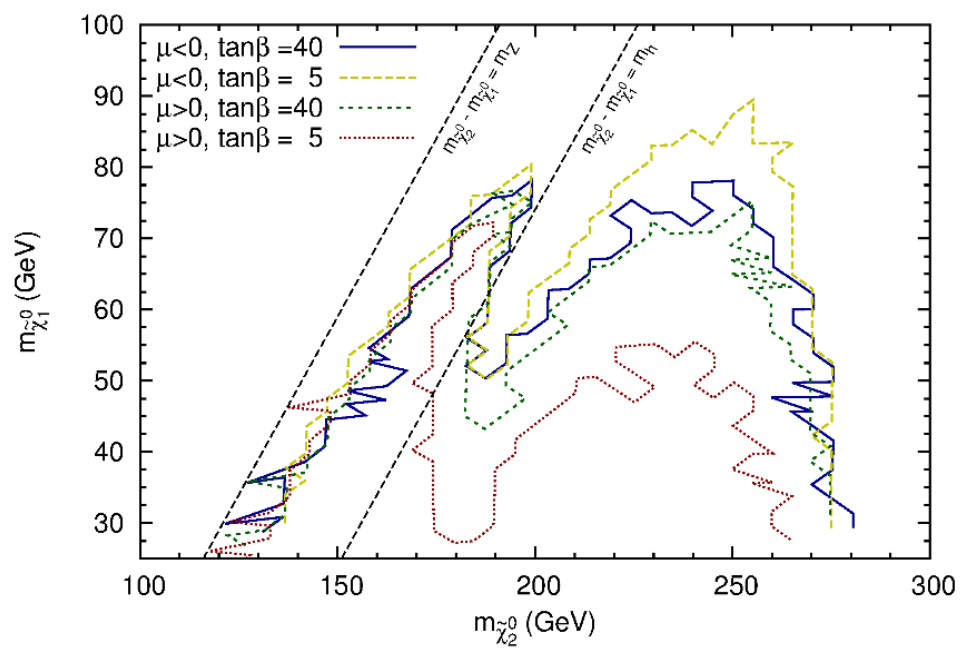

Figure 7. Reinterpreted ATLAS limit [3] for the Higgsino-like $\widetilde{\chi}_{2,3}^{0}$ case, displayed in the $m_{\widetilde{\chi}_{2}^{0}}-m_{\widetilde{\chi}_{1}^{0}}$ ) plane for different values of $\tan \beta, \operatorname{sgn}(\mu)$. See the text for details.

on electroweak production of Higgsinos and their decay. In particular, the production cross sections have no dependence on any squark masses, in contrast to the wino case, for which $t$-channel squark exchange decouples only very slowly and can be relevant even for very heavy squarks [60]. In contrast to the wino case the Higgsinos have only small couplings with first and second generation quarks and squarks rendering such contributions negligible.

\section{LHC limits}

As discussed in the last section, the scenario under consideration can best be searched for at the LHC in the $W Z$ channel, where both CMS and ATLAS have performed different searches using the full dataset available at $8 \mathrm{TeV}[1-3]$. The most stringent limit available is deduced from the three-leptons plus missing energy search performed by ATLAS [3]. In the relevant signal region three leptons have to be identified, where two of them have to be of the same flavour and of different sign (SF-OS). The resulting event sample is further divided into 16 bins with different invariant mass cuts for the SF-OS pair, different cuts on the transverse mass $m_{T}$ and/or different cuts on the transverse missing energy $\mathbb{E}_{T}$. Final event numbers are found to be in good agreement with Standard Model predictions. Interpreting the resulting limits in a pure wino scenario ${ }^{4}$ with $\operatorname{BR}\left(\widetilde{\chi}_{2}^{0} \rightarrow Z \widetilde{\chi}_{1}^{0}\right)=100 \%$ (as explained above) ATLAS sets bounds up to $m_{\widetilde{\chi}_{1}^{ \pm}}=m_{\widetilde{\chi}_{2}^{0}} \gtrsim 350 \mathrm{GeV}$ for a massless neutralino. Furthermore, the ATLAS collaboration interprets the search in the $M_{2}$ - $\mu$-plane of a pMSSM scenario with decoupled sfermions, a bino of $M_{1}=50 \mathrm{GeV}$ and $\tan \beta=10$. Here, for $M_{2} \gg \mu$ a limit of $\mu \gtrsim 230 \mathrm{GeV}$ is derived. We want to reinterpret this limit in the light neutralino scenario discussed above, where we vary both $M_{1}$ and $\tan \beta$ (besides $\mu$ and $M_{2}$ ).

\footnotetext{
${ }^{4}$ In this scenario the sfermions are decoupled at $m_{\tilde{f}}=5 \mathrm{TeV}$ as listed on HepData [62].
} 
In our Monte Carlo study we use Herwig++ [63] for event simulation and rescale the obtained LO rates with NLO K-factors obtained from Prospino 2 [61]. Furthermore, we use the powerful CheckMate [64] framework for detector simulation (where a tuned version of Delphes $3[65]$ is used internally), analysis and statistical evaluation. First, we carefully verified that the three-leptons plus missing energy analysis implemented in CheckMate yields limits for the pure-wino scenario and the pMSSM scenario which are in good agreement with the ones published by ATLAS. Second, we evaluate those limits in the benchmark scenarios motivated in section 3, i.e. we translate the ATLAS limits into the $M_{1}-\mu$ plane for $\tan \beta=5,40$ and $\mu \gtrless 0$. As discussed in section 3 , for $\mu>0, \tan \beta=5$ gives a conservative limit while $\tan \beta=40$ gives a limit in the plateau shown in figure 4 . On the contrary, for $\mu<0$, the large $\tan \beta$ case corresponds to a conservative scenario. As also discussed in section 3 decoupling $M_{2}$ yields a conservative bound and thus we set $M_{2}=1 \mathrm{TeV}$.

Resulting limits are shown in figure 7 projected on the plane of the physical masses $m_{\widetilde{\chi}_{1}^{0}}$ vs. $m_{\widetilde{\chi}_{2}^{0}}$. For a lightest neutralino of mass $m_{\widetilde{\chi}_{1}^{0}}=35 \mathrm{GeV}, \tan \beta=5$ and $\mu>0$ we find a limit of $m_{\widetilde{\chi}_{2}^{0}} \lesssim 120, m_{\widetilde{\chi}_{2}^{0}} \gtrsim 260 \mathrm{GeV}$. The lower limit is a consequence of the kinematic boundary between on- and off-shell decays at $m_{\widetilde{\chi}_{2}^{0}}-m_{\widetilde{\chi}_{1}^{0}}=m_{Z}$. In the regime of purely off-shell decays of the $\widetilde{\chi}_{2}^{0}$ various decay modes compete and the ATLAS limit vanishes. Furthermore, as discussed in [22], in this regime branching ratios can still strongly depend on the scale and details of the "decoupled" sfermions, thus conservative exclusion limits are difficult to deduce. For $\tan \beta=40$ and $\mu<0$ the upper limits extends up to $m_{\widetilde{\chi}_{2}^{0}} \gtrsim 280 \mathrm{GeV}$. At the same time in this case larger values of $m_{\widetilde{\chi}_{1}^{0}}$ can be excluded. For all scenarios studied exclusion limits drop sharply at the kinematical threshold $m_{\widetilde{\chi}_{2}^{0}}-m_{\widetilde{\chi}_{1}^{0}}=$ $m_{h}$. In this small corner of the parameter space limits from $W h$ searches might become relevant $[24,59,60]$.

Finally we present the limits of our reinterpretation in the phenomenologically interesting $\widetilde{\chi}_{1}^{0}$ vs. $|\mu|$ plane, i.e. in the plane where constraints from the thermal relic abundance were discussed in figure 1 . Results are shown in the upper/lower panel of figure 8 for $\tan \beta=5 / 40$; on the left and right for $\mu>0$ and $\mu<0$ respectively. Regions excluded by LHC searches are shaded in yellow and as in figure 1 regions yielding the correct relic abundance just from neutralino DM are shown in blue, while regions where the abundance of the $\widetilde{\chi}_{1}^{0}$ could contribute to the overall DM abundance are shown in red. For all choices of $\tan \beta$ and $\operatorname{sgn}(\mu)$ large parts of the $Z$-resonance regions are excluded. More precisely, at the $Z$-resonance we find $\mu \gtrsim 250 \mathrm{GeV}$, apart from a small strip around $\left(|\mu| \approx m_{\widetilde{\chi}_{2}^{0}}\right)-m_{\widetilde{\chi}_{1}^{0}}=m_{h}$ for $\mu>0$ and in general for very small values of $\mu$ (below the $\widetilde{\chi}_{2}^{0} \rightarrow Z \widetilde{\chi}_{1}^{0}$ threshold). However, at least for $\mu>0$, here the limit from $\operatorname{BR}(h \rightarrow$ invisible) shown in eq. (2.20) become relevant, excluding the points shown in grey. Combining these limits for $\tan \beta=40$ we find

$$
m_{\widetilde{\chi}_{1}^{0}} \gtrsim 40 \mathrm{GeV} \quad[\mu>0, \tan \beta=40] .
$$

In the more conservative scenario with $\tan \beta=5$ the bound is somewhat weaker and we find

$$
m_{\widetilde{\chi}_{1}^{0}} \gtrsim 37 \mathrm{GeV} \quad[\mu>0, \tan \beta=5]
$$



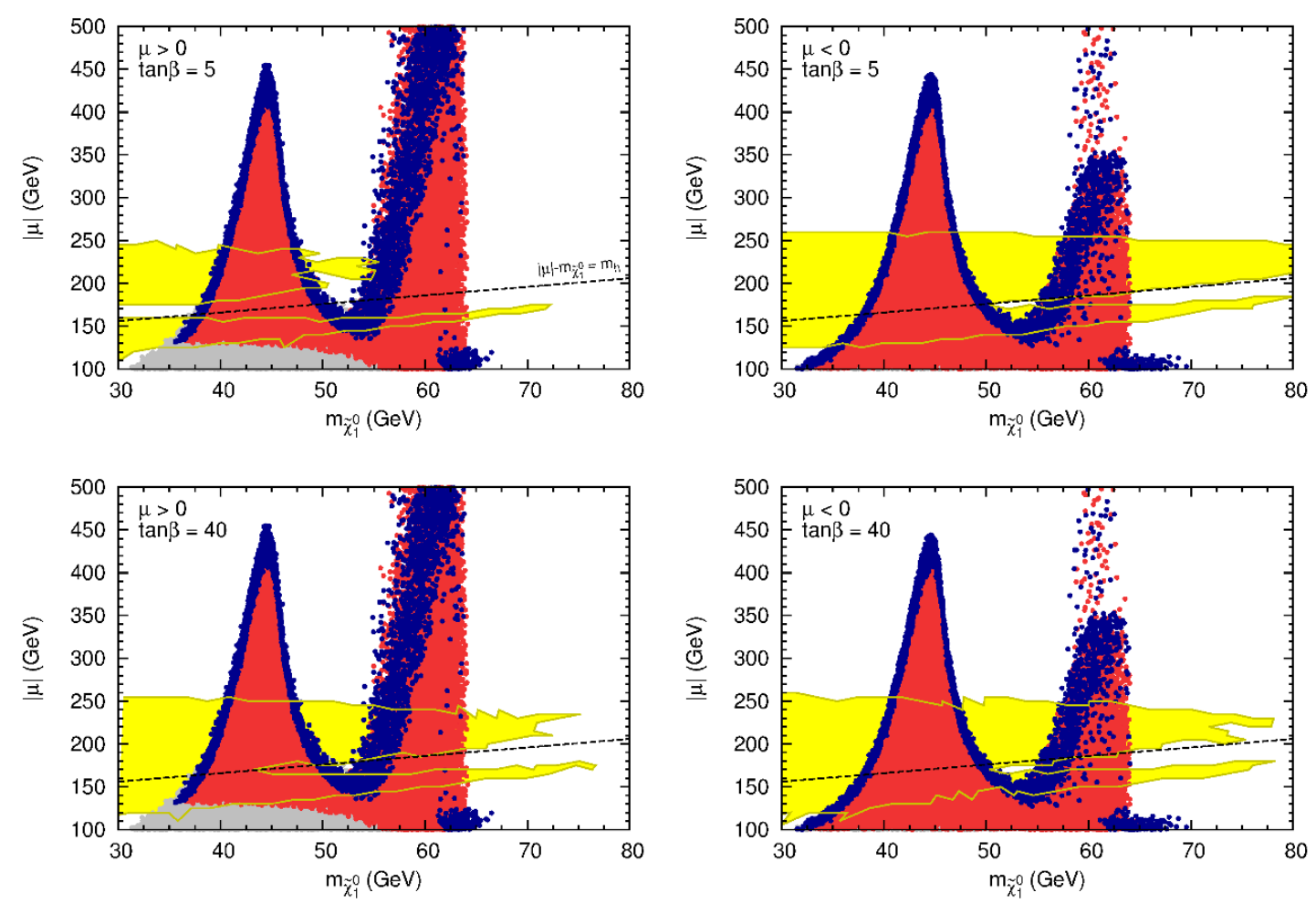

Figure 8. Reinterpreted ATLAS limit, displayed in the $m_{\widetilde{\chi}_{1}^{0}}-|\mu|$ plane of figure 1 for different values of $\tan \beta, \operatorname{sgn}(\mu)$. Color code as in figure 1 . The yellow regions are excluded by ATLAS three leptons plus missing energy search [3].

For $\mu<0$ the constraint from the relic abundance combined with the LEP limit on charginos still yields the strongest bound as very small values of $\mu$ cannot be excluded. As we have already seen in section 2.1 here,

$$
m_{\widetilde{\chi}_{1}^{0}} \gtrsim 30 \mathrm{GeV} \quad[\mu<0] .
$$

Also the $h$-resonance region can partly be excluded already. Precise limits can be read of figure 8. Noteworthy, this region extents to very large values of $\mu$, beyond the scope of even the high energy LHC.

\section{Conclusions}

In this work, we have studied light neutralino Dark Matter in the MSSM within frameworks where all sfermions are heavy. Interestingly, this feature of the spectrum is shared by both 'natural SUSY' and 'mini split' scenarios. Under the assumption that the neutralino is a standard thermal relic, CMB measurements of the DM abundance translate into specific requirements the spectrum must fulfill. Since, in our case, sfermions play no role in the computation of the DM relic density, these bounds must be satisfied by the neutralino/chargino sector of the MSSM alone. The generic requirement is that Higgsinos are relatively light, such that the lightest neutralino can couple to $Z$ or $h$ through a nonnegligible Higgsino component and thus efficiently annihilates. This condition is strongly 
relaxed if the neutralino mass approaches the conditions for a resonant enhancement of the annihilation cross section: $m_{\widetilde{\chi}_{1}^{0}} \simeq m_{Z} / 2$ or $m_{\widetilde{\chi}_{1}^{0}} \simeq m_{h} / 2$. In such a case, Higgsinos can be as heavy as $450 \mathrm{GeV}$ and $1.2 \mathrm{TeV}$ respectively. This parameter space, depicted in figure 1, can be hardly tested by direct and indirect DM search experiments because of a suppression of the relevant cross sections in correspondence of the resonances, as shown in figure 3. In contrast, LHC experiments have the potential to partly test our scenario searching for production of Higgsino-like charginos and neutralinos, followed by decays to $W Z$ and the LSP. In fact, the remarkable sensitivity reached by the LHC experiments in the search for purely electroweakly interacting new particles allows us to directly test the electroweak sector of supersymmetric models without the need of assumptions on the strongly-interacting superpartners.

In section 4, we have presented the results of our reinterpretation of an ATLAS three leptons plus missing energy search. In figure 8, we have shown that LHC experiments can set non-trivial constraints on the light neutralino parameter space already with the data collected at $\sqrt{s}=8 \mathrm{TeV}$. In particular, we have seen that the current limit only leaves uncovered the case of a neutralino mass lying close to the resonances (at about $5 \mathrm{GeV}$ or less), as well as the corners of the parameter space corresponding to the kinematical thresholds $|\mu|-m_{\widetilde{\chi}_{1}^{0}}=m_{Z}, m_{h}$, where the three leptons searches loose sensitivity. Combining with further channels, such as di-leptons plus missing energies, as well as searches for $W h$ events, could further reduce the uncovered corners.

The exercise we have performed demonstrates once more that LHC searches for electroweakly interacting SUSY particles can be successfully interpreted as indirect searches for supersymmetric Dark Matter at collider (especially in combination with the relic density constraints), often resulting in more stringent limits than those set by Dark Matter experiments themselves.

\section{Acknowledgments}

We thank Jamie Tattersall for providing us with a private version of CheckMate. We would also like to thank Andreas Papaefstathiou for useful discussions and help with Herwig++. We also thank Ulrich Ellwanger and Guillaume Drieu La Rochelle for valuable discussions. JML was supported by the European Commission through the "LHCPhenoNet" Initial Training Network PITN-GA-2010-264564. The research of TO is supported by Grantsin-Aid for Scientific Research on Innovative Areas Unification and Development of the Neutrino Science Frontier Number 26105503.

\section{A Neutralino masses and mixing}

The neutralino mass term in the MSSM Lagrangian

$$
\mathscr{L}=-\frac{1}{2}\left(\overline{\psi^{c}}\right)_{\alpha}\left(\mathcal{M}_{\widetilde{\chi}^{0}}\right)_{\alpha \beta} \psi_{\beta},
$$


is given with the following symmetric matrix,

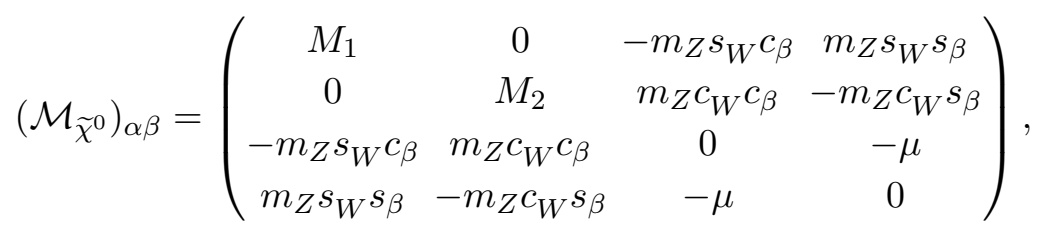

in the gauge-interaction basis $\psi_{\alpha}=\left(\widetilde{B}, \widetilde{W}^{0}, \widetilde{H}_{d}^{0}, \widetilde{H}_{u}^{0}\right)$, where $s_{W}=\sin \theta_{W}, c_{W}=\cos \theta_{W}$, $s_{\beta}=\sin \beta$, and $c_{\beta}=\cos \beta$. Although we diagonalize this mass matrix (including additional higher order radiative corrections) numerically to obtain the mass eigenvalues $m_{\widetilde{\chi}_{i}^{0}}$ and the mass eigenstates $\tilde{\chi}_{i}^{0}=N_{i \alpha} \psi_{\alpha}$ with the mixing matrix $N_{i \alpha}$, we derive analytic and approximated expressions to grasp the trend of the numerical results.

First, we separate $\mathcal{M}_{\widetilde{\chi}^{0}}$ into the zero-th order part $\mathcal{M}_{0}$ and the perturbation $\delta \mathcal{M}$ as $\mathcal{M}_{\widetilde{\chi}^{0}}=\mathcal{M}_{0}+\delta \mathcal{M}$. They are defined as

$$
\begin{aligned}
\left(\mathcal{M}_{0}\right)_{\alpha \beta} & =\left(\begin{array}{cccc}
M_{1} & 0 & 0 & 0 \\
0 & M_{2} & 0 & 0 \\
0 & 0 & 0 & -\mu \\
0 & 0 & -\mu & 0
\end{array}\right), \\
(\delta \mathcal{M})_{\alpha \beta} & =\left(\begin{array}{cccc}
0 & 0 & -m_{Z} s_{W} c_{\beta} & m_{Z} s_{W} s_{\beta} \\
0 & 0 & m_{Z} c_{W} c_{\beta} & -m_{Z} c_{W} s_{\beta} \\
-m_{Z} s_{W} c_{\beta} & m_{Z} c_{W} c_{\beta} & 0 & 0 \\
m_{Z} s_{W} s_{\beta} & -m_{Z} c_{W} s_{\beta} & 0 & 0
\end{array}\right) .
\end{aligned}
$$

The zero-th order part $\mathcal{M}_{0}$ can be diagonalized with the zero-th order mixing matrix $N_{i \alpha}^{(0)}$

$$
N_{i \alpha}^{(0)}=\left(\begin{array}{cccc}
1 & 0 & 0 & 0 \\
0 & 0 & -\frac{1}{\sqrt{2}} & \frac{1}{\sqrt{2}} \\
0 & 0 & -\frac{1}{\sqrt{2}} & -\frac{1}{\sqrt{2}} \\
0 & -1 & 0 & 0
\end{array}\right)
$$

and the mass eigenvalues at the zero-th order are given as ${ }^{5}$

$$
m_{\widetilde{\chi}_{1}^{0}}^{(0)}=M_{1}, \quad m_{\widetilde{\chi}_{2}^{0}}^{(0)}=\mu, \quad m_{\widetilde{\chi}_{3}^{0}}^{(0)}=-\mu, \quad m_{\widetilde{\chi}_{4}^{0}}^{(0)}=M_{2} .
$$

Since we assume $M_{1} \ll|\mu| \ll M_{2}$ in our scenario, here we arrange the ordering of the mass eigenstates as described with eq. (A.5).

Next, we take into account the effect from the perturbation part $\delta \mathcal{M}$. This perturbation is valid, if the condition $(\delta \mathcal{M})_{i j} \ll\left|m_{\widetilde{\chi}_{i}^{0}}^{(0)}-m_{\widetilde{\chi}_{j}^{0}}^{(0)}\right|$ is fulfilled, where $(\delta \mathcal{M})_{i j}$ is the

\footnotetext{
${ }^{5}$ At the zero-th order, $\widetilde{\chi}_{2}^{0}$ and $\widetilde{\chi}_{3}^{0}$ are degenerate in physical mass. The second lightest state and the third lightest state can be identified only after taking the radiative corrections (and the perturbation) into account. In the case of $\mu>0$, we identify the state with a mass of $\mu$ as a would-be $\widetilde{\chi}_{2}^{0}$, and that with $-\mu$ as a would-be $\widetilde{\chi}_{3}^{0}$. In the case of $\mu<0$, the ordering becomes opposite, i.e., $m_{\tilde{\chi}_{2}^{0}}^{(0)}=-\mu(>0)$ and $m_{\widetilde{\chi}_{3}^{0}}^{(0)}=\mu(<0)$. Therefore, the mixing matrix for the $\mu<0$ case can be obtained by exchanging $N_{2 \alpha}$ and $N_{3 \alpha}$ in eq. (A.7).
} 
perturbation part in the zero-th order mass eigenbasis, i.e., $(\delta \mathcal{M})_{i j}=N_{i \alpha}^{(0)}(\delta \mathcal{M})_{\alpha \beta} N_{\beta j}^{(0) \top}$. In the $M_{2}$ decoupling limit, the perturbation terms are proportional to $m_{Z} s_{W}$, which is sufficiently smaller than the difference between two mass eigenvalues, which is typically $|\mu|$. After including the first order perturbation, the neutralino mixings $N_{i \alpha}$ result in the following:

$$
N_{i \alpha}^{(0+1)}=\left(\begin{array}{cccc}
1 & 0 & \frac{m_{Z} s_{W}}{\mu}\left(s_{\beta}+c_{\beta} \frac{M_{1}}{\mu}\right) & -\frac{m_{Z} s_{W}}{\mu}\left(c_{\beta}+s_{\beta} \frac{M_{1}}{\mu}\right) \\
\frac{m_{Z} s_{W}\left(s_{\beta}+c_{\beta}\right)}{\sqrt{2} \mu}\left(1+\frac{M_{1}}{\mu}\right) & 0 & -\frac{1}{\sqrt{2}} & \frac{1}{\sqrt{2}} \\
\frac{m_{Z} s_{W}\left(s_{\beta}-c_{\beta}\right)}{\sqrt{2} \mu}\left(1-\frac{M_{1}}{\mu}\right) & 0 & -\frac{1}{\sqrt{2}} & -\frac{1}{\sqrt{2}} \\
0 & -1 & 0 & 0
\end{array}\right)
$$

Here, we expand the matrix elements in powers of $M_{1} / \mu$ and keep the terms up to the first order. The approximate results fit well with the numerical results evaluated by SuSpect. Although the mass eigenvalues do not get the correction at the first order of this perturbation, they are affected by the radiative corrections [66], which are larger than the perturbations. For the analytic expressions of the neutralino masses and mixings in various cases, see e.g. ref. [67].

Open Access. This article is distributed under the terms of the Creative Commons Attribution License (CC-BY 4.0), which permits any use, distribution and reproduction in any medium, provided the original author(s) and source are credited.

\section{References}

[1] ATLAS collaboration, Search for direct production of charginos, neutralinos and sleptons in final states with two leptons and missing transverse momentum in pp collisions at $\sqrt{s}=8 \mathrm{TeV}$ with the ATLAS detector, JHEP 05 (2014) 071 [arXiv:1403.5294] [INSPIRE].

[2] CMS collaboration, Searches for electroweak production of charginos, neutralinos and sleptons decaying to leptons and $W, Z$ and Higgs bosons in pp collisions at 8 TeV, Eur. Phys. J. C 74 (2014) 3036 [arXiv:1405.7570] [INSPIRE].

[3] ATLAS collaboration, Search for direct production of charginos and neutralinos in events with three leptons and missing transverse momentum in $\sqrt{s}=8 \mathrm{TeV}$ pp collisions with the ATLAS detector, JHEP 04 (2014) 169 [arXiv:1402.7029] [INSPIRE].

[4] L. Calibbi, J.M. Lindert, T. Ota and Y. Takanishi, Cornering light neutralino dark matter at the LHC, JHEP 10 (2013) 132 [arXiv:1307.4119] [INSPIRE].

[5] L. Calibbi, J.M. Lindert, T. Ota and Y. Takanishi, A lower bound on light neutralino dark matter from LHC data, arXiv: 1405.3884 [INSPIRE].

[6] A. Arbey, M. Battaglia and F. Mahmoudi, Supersymmetry with light dark matter confronting the recent CDMS and LHC results, Phys. Rev. D 88 (2013) 095001 [arXiv:1308.2153] [INSPIRE].

[7] G. Belanger et al., LHC constraints on light neutralino dark matter in the MSSM, Phys. Lett. B 726 (2013) 773 [arXiv:1308.3735] [INSPIRE]. 
[8] K. Hagiwara, S. Mukhopadhyay and J. Nakamura, $10 \mathrm{GeV}$ neutralino dark matter and light stau in the MSSM, Phys. Rev. D 89 (2014) 015023 [arXiv: 1308.6738] [INSPIRE].

[9] C. Boehm, P.S.B. Dev, A. Mazumdar and E. Pukartas, Naturalness of light neutralino dark matter in pMSSM after LHC, XENON100 and Planck data, JHEP 06 (2013) 113 [arXiv:1303.5386] [INSPIRE].

[10] G. Bélanger, S. Biswas, C. Boehm and B. Mukhopadhyaya, Light neutralino dark matter in the MSSM and its implication for LHC searches for staus, JHEP 12 (2012) 076 [arXiv: 1206.5404] [INSPIRE].

[11] D. Hooper and T. Plehn, Supersymmetric dark matter: how light can the LSP be?, Phys. Lett. B 562 (2003) 18 [hep-ph/0212226] [INSPIRE].

[12] G. Bélanger, F. Boudjema, A. Pukhov and S. Rosier-Lees, A lower limit on the neutralino mass in the MSSM with nonuniversal gaugino masses, hep-ph/0212227 [INSPIRE].

[13] A. Bottino, N. Fornengo and S. Scopel, Light relic neutralinos, Phys. Rev. D 67 (2003) 063519 [hep-ph/0212379] [INSPIRE].

[14] H.K. Dreiner et al., Mass bounds on a very light neutralino, Eur. Phys. J. C 62 (2009) 547 [arXiv:0901.3485] [INSPIRE].

[15] H.K. Dreiner et al., Rare meson decays into very light neutralinos, Phys. Rev. D 80 (2009) 035018 [arXiv: 0905. 2051] [INSPIRE].

[16] M. Cahill-Rowley et al., Complementarity of dark matter searches in the pMSSM, arXiv:1405.6716 [INSPIRE].

[17] N. Arkani-Hamed, A. Delgado and G.F. Giudice, The well-tempered neutralino, Nucl. Phys. B 741 (2006) 108 [hep-ph/0601041] [INSPIRE].

[18] J. Bramante, A. Delgado, F. Elahi, A. Martin and B. Ostdiek, Catching sparks from well-forged neutralinos, arXiv:1408.6530 [INSPIRE].

[19] G.F. Giudice and A. Romanino, Split supersymmetry, Nucl. Phys. B 699 (2004) 65 [Erratum ibid. B 706 (2005) 65-89] [hep-ph/0406088] [INSPIRE].

[20] N. Arkani-Hamed and S. Dimopoulos, Supersymmetric unification without low energy supersymmetry and signatures for fine-tuning at the LHC, JHEP 06 (2005) 073 [hep-th/0405159] [INSPIRE].

[21] A. Arvanitaki, N. Craig, S. Dimopoulos and G. Villadoro, Mini-split, JHEP 02 (2013) 126 [arXiv: 1210.0555] [INSPIRE].

[22] A. Bharucha, S. Heinemeyer and F. von der Pahlen, Direct chargino-neutralino production at the LHC: interpreting the exclusion limits in the complex MSSM, Eur. Phys. J. C 73 (2013) 2629 [arXiv: 1307.4237] [INSPIRE].

[23] S. Gori, S. Jung and L.-T. Wang, Cornering electroweakinos at the LHC, JHEP 10 (2013) 191 [arXiv: 1307.5952] [INSPIRE].

[24] T. Han, S. Padhi and S. Su, Electroweakinos in the light of the Higgs boson, Phys. Rev. D 88 (2013) 115010 [arXiv:1309.5966] [INSPIRE].

[25] P. Schwaller and J. Zurita, Compressed electroweakino spectra at the LHC, JHEP 03 (2014) 060 [arXiv: 1312.7350] [INSPIRE].

[26] Z. Han, G.D. Kribs, A. Martin and A. Menon, Hunting quasidegenerate Higgsinos, Phys. Rev. D 89 (2014) 075007 [arXiv: 1401.1235] [INSPIRE]. 
[27] C. Han, L. Wu, J.M. Yang, M. Zhang and Y. Zhang, A new approach for detecting compressed bino/wino at the LHC, arXiv:1409.4533 [INSPIRE].

[28] T.A.W. Martin and D. Morrissey, Electroweakino constraints from LHC data, arXiv:1409.6322 [INSPIRE].

[29] T. Nihei, L. Roszkowski and R. Ruiz de Austri, Exact cross-sections for the neutralino WIMP pair annihilation, JHEP 03 (2002) 031 [hep-ph/0202009] [INSPIRE].

[30] H.E. Haber and G.L. Kane, The search for supersymmetry: probing physics beyond the standard model, Phys. Rept. 117 (1985) 75 [INSPIRE].

[31] M. Drees and M.M. Nojiri, The neutralino relic density in minimal $N=1$ supergravity, Phys. Rev. D 47 (1993) 376 [hep-ph/9207234] [INSPIRE].

[32] A. Bottino, F. Donato, N. Fornengo and S. Scopel, Indirect signals from light neutralinos in supersymmetric models without gaugino mass unification, Phys. Rev. D 70 (2004) 015005 [hep-ph/0401186] [INSPIRE].

[33] A. Bottino, F. Donato, N. Fornengo and S. Scopel, Interpreting the recent results on direct search for dark matter particles in terms of relic neutralino, Phys. Rev. D 78 (2008) 083520 [arXiv:0806.4099] [INSPIRE].

[34] N. Fornengo, S. Scopel and A. Bottino, Discussing direct search of dark matter particles in the Minimal Supersymmetric extension of the standard model with light neutralinos, Phys. Rev. D 83 (2011) 015001 [arXiv: 1011.4743] [INSPIRE].

[35] L. Calibbi, T. Ota and Y. Takanishi, Light neutralino in the MSSM: a playground for dark matter, flavor physics and collider experiments, JHEP 07 (2011) 013 [arXiv:1104.1134] [INSPIRE].

[36] CMS collaboration, Search for neutral MSSM Higgs bosons decaying to a pair of $\tau$ leptons in pp collisions, JHEP 10 (2014) 160 [arXiv:1408.3316] [INSPIRE].

[37] P. Bechtle, S. Heinemeyer, O. Stål, T. Stefaniak and G. Weiglein, Probing the standard model with Higgs signal rates from the Tevatron, the LHC and a future ILC, arXiv:1403.1582 [INSPIRE].

[38] LHCb collaboration, First evidence for the decay $B_{s}^{0} \rightarrow \mu^{+} \mu^{-}$, Phys. Rev. Lett. 110 (2013) 021801 [arXiv: 1211.2674] [INSPIRE].

[39] A. Djouadi, J.-L. Kneur and G. Moultaka, SuSpect: a Fortran code for the supersymmetric and Higgs particle spectrum in the MSSM, Comput. Phys. Commun. 176 (2007) 426 [hep-ph/0211331] [INSPIRE].

[40] A. Djouadi, M.M. Muhlleitner and M. Spira, Decays of supersymmetric particles: the program SUSY-HIT (SUspect-SdecaY-HDECAY-InTerface), Acta Phys. Polon. B 38 (2007) 635 [hep-ph/0609292] [INSPIRE].

[41] G. Bélanger, F. Boudjema, A. Pukhov and A. Semenov, MicrOMEGAs_3: a program for calculating dark matter observables, Comput. Phys. Commun. 185 (2014) 960 [arXiv: 1305.0237] [INSPIRE].

[42] G. Bélanger, F. Boudjema, A. Pukhov and A. Semenov, MicrOMEGAs 2.0: a program to calculate the relic density of dark matter in a generic model, Comput. Phys. Commun. 176 (2007) 367 [hep-ph/0607059] [INSPIRE]. 
[43] G. Bélanger, F. Boudjema, A. Pukhov and A. Semenov, MicrOMEGAs: version 1.3, Comput. Phys. Commun. 174 (2006) 577 [hep-ph/0405253] [INSPIRE].

[44] G. Bélanger, F. Boudjema, A. Pukhov and A. Semenov, MicrOMEGAs: a program for calculating the relic density in the MSSM, Comput. Phys. Commun. 149 (2002) 103 [hep-ph/0112278] [INSPIRE].

[45] Planck collaboration, P.A.R. Ade et al., Planck 2013 results. XVI. Cosmological parameters, Astron. Astrophys. (2014) [arXiv:1303.5076] [INSPIRE].

[46] J.R. Ellis, J.M. Frere, J.S. Hagelin, G.L. Kane and S.T. Petcov, Search for neutral gauge fermions in $e^{+} e^{-}$annihilation, Phys. Lett. B 132 (1983) 436 [InSPIRE].

[47] A. Bartl, H. Fraas and W. Majerotto, Production and decay of neutralinos in $e^{+} e^{-}$ annihilation, Nucl. Phys. B 278 (1986) 1 [InSPIRE].

[48] R. Barbieri, G. Gamberini, G.F. Giudice and G. Ridolfi, Neutralino production at (and close to) the $Z^{0}$ peak, Phys. Lett. B 195 (1987) 500 [INSPIRE].

[49] ALEPH, DELPHI, L3, OPAL, SLD, LEP Electroweak Working Group, SLD Electroweak Group, SLD Heavy Flavour Group collaboration, S. Schael et al., Precision electroweak measurements on the $Z$ resonance, Phys. Rept. 427 (2006) 257 [hep-ex/0509008] [INSPIRE].

[50] P. Bechtle, O. Brein, S. Heinemeyer, G. Weiglein and K.E. Williams, HiggsBounds: confronting arbitrary higgs sectors with exclusion bounds from LEP and the Tevatron, Comput. Phys. Commun. 181 (2010) 138 [arXiv:0811.4169] [INSPIRE].

[51] P. Bechtle, O. Brein, S. Heinemeyer, G. Weiglein and K.E. Williams, HiggsBounds 2.0.0: confronting neutral and charged Higgs sector predictions with exclusion bounds from LEP and the Tevatron, Comput. Phys. Commun. 182 (2011) 2605 [arXiv:1102.1898] [InSPIRE].

[52] P. Bechtle et al., HiggsBounds-4: improved tests of extended Higgs sectors against exclusion bounds from LEP, the Tevatron and the LHC, Eur. Phys. J. C 74 (2014) 2693 [arXiv: 1311.0055] [INSPIRE].

[53] P. Bechtle, S. Heinemeyer, O. Stål, T. Stefaniak and G. Weiglein, HiggsSignals: confronting arbitrary Higgs sectors with measurements at the Tevatron and the LHC, Eur. Phys. J. C 74 (2014) 2711 [arXiv: 1305.1933] [inSPIRE].

[54] K. Griest and H.E. Haber, Invisible decays of Higgs bosons in supersymmetric models, Phys. Rev. D 37 (1988) 719 [inSPIRE].

[55] LUX collaboration, D.S. Akerib et al., First results from the LUX dark matter experiment at the Sanford Underground Research Facility, Phys. Rev. Lett. 112 (2014) 091303 [arXiv: 1310.8214] [INSPIRE].

[56] Fermi-LAT collaboration, M. Ackermann et al., Constraining dark matter models from a combined analysis of Milky Way satellites with the Fermi Large Area Telescope, Phys. Rev. Lett. 107 (2011) 241302 [arXiv: 1108.3546] [INSPIRE].

[57] C. Han et al., Probing light higgsinos in natural SUSY from monojet signals at the LHC, JHEP 02 (2014) 049 [arXiv:1310.4274] [INSPIRE].

[58] M. Low and L.-T. Wang, Neutralino dark matter at 14 TeV and $100 \mathrm{TeV}$, JHEP 08 (2014) 161 [arXiv: 1404.0682] [INSPIRE]. 
[59] H. Baer, V. Barger, A. Lessa, W. Sreethawong and X. Tata, Wh plus missing- $E_{T}$ signature from gaugino pair production at the LHC, Phys. Rev. D 85 (2012) 055022 [arXiv:1201.2949] [INSPIRE].

[60] A. Papaefstathiou, K. Sakurai and M. Takeuchi, Higgs boson to di-tau channel in chargino-neutralino searches at the LHC, JHEP 08 (2014) 176 [arXiv: 1404.1077] [INSPIRE].

[61] W. Beenakker et al., The Production of charginos/neutralinos and sleptons at hadron colliders, Phys. Rev. Lett. 83 (1999) 3780 [Erratum ibid. 100 (2008) 029901] [hep-ph/9906298] [INSPIRE].

[62] http://hepdata.cedar.ac.uk/view/ins1282905.

[63] J. Bellm et al., HERWIG++2.7 release note, arXiv:1310.6877 [INSPIRE].

[64] M. Drees, H. Dreiner, D. Schmeier, J. Tattersall and J.S. Kim, CheckMATE: confronting your favourite new physics model with LHC data, arXiv:1312.2591 [INSPIRE].

[65] DELPHES 3 collaboration, J. de Favereau et al., DELPHES 3, a modular framework for fast simulation of a generic collider experiment, JHEP 02 (2014) 057 [arXiv:1307.6346] [INSPIRE].

[66] T. Fritzsche and W. Hollik, Complete one loop corrections to the mass spectrum of charginos and neutralinos in the MSSM, Eur. Phys. J. C 24 (2002) 619 [hep-ph/0203159] [InSPIRE].

[67] A. Bartl, H. Fraas, W. Majerotto and N. Oshimo, The neutralino mass matrix in the minimal supersymmetric model, Phys. Rev. D 40 (1989) 1594 [INSPIRE]. 